\title{
Interaction of the Particle with the String in Pole-Dipole Ap- proximation
}

\author{
Milovan Vasilić ${ }^{1, *}$ and Marko Vojinović ${ }^{1, * *}$ \\ ${ }^{1}$ Institute of Physics, P.O.Box 57, 11001 Belgrade, Serbia
}

\begin{abstract}
Key words Equations of motion, Regge trajectories, strings, boundary conditions, Papapetrou method PACS 04.20.-q, 04.40.-b, 11.25.-w

Within the framework of generalized Papapetrou method, we derive the effective equations of motion for a string with two particles attached to its ends, along with appropriate boundary conditions. The equations of motion are the usual Nambu-Goto-like equations, while boundary conditions turn out to be equations of motion for the particles at the string ends. The form of those equations is discussed, and they are explicitly solved for a particular case of a straight-line string rotating around its center. From this solution we obtain the correction terms to the $J \propto E^{2}$ law describing Regge trajectories, due to nonzero angular momenta of the particles.
\end{abstract}

Copyright line will be provided by the publisher

\section{Introduction}

The interest in studying extended objects in high energy physics began with the observation that meson resonances could be viewed as rotating relativistic strings [1, 2]. This model provided a successful explanation of Regge trajectories. Nevertheless, the model assumes that the two quarks attached to the string have zero mass and zero angular momentum. The purpose of this paper is to take into account small mass and small angular momentum of the two particles, thereby giving a correction term in $J \propto E^{2}$ law describing Regge trajectories.

In order to do so, one needs to derive the equations of motion for the string with particles attached to its ends. The general method that lends itself for doing this was developed in [3, 4], and represents the generalization of the Mathisson-Papapetrou method [5, 6] to include extended objects.

Our conventions are the same as in [4]. Greek indices $\mu, \nu, \ldots$ are the spacetime indices, and run over $0,1,2,3$. Latin indices $a, b, \ldots$ are the world sheet indices and run over 0,1 . The Latin indices $i, j, \ldots$ refer to the world sheet boundary and take a single value 0 . The coordinates of spacetime, world sheet and world sheet boundary are denoted by $x^{\mu}, \xi^{a}$ and $\lambda^{i}$, respectively. The corresponding metric tensors are denoted by $g_{\mu \nu}(x), \gamma_{a b}(\xi)$ and $h_{i j}(\lambda)$. The signature convention is defined by $\operatorname{diag}(-,+, \ldots,+)$, and the indices are raised by the inverse metrics $g^{\mu \nu}, \gamma^{a b}$ and $h^{i j}$.

\section{Equations of motion}

The basic starting point of the analysis is the covariant conservation of the symmetric stress-energy tensor

$$
\nabla_{\nu} T^{\mu \nu}=0 .
$$

The stress-energy tensor is written as a sum of two terms,

$$
T^{\mu \nu}=T_{\mathrm{s}}^{\mu \nu}+T_{\mathrm{p}}^{\mu \nu},
$$

* E-mail: mvasilic@phy.bg.ac.yu

** Corresponding author E-mail: vmarko@phy.bg.ac.yu, Phone: +381 113713147 , Fax: +381 113162190 
where

$$
\begin{aligned}
& T_{\mathrm{s}}^{\mu \nu}=\int_{\mathcal{M}} d^{2} \xi \sqrt{-\gamma} B_{\mathrm{s}}^{\mu \nu} \frac{\delta^{(4)}(x-z)}{\sqrt{-g}}, \\
& T_{\mathrm{p}}^{\mu \nu}=\int_{\partial \mathcal{M}} d \lambda \sqrt{-h}\left(B_{\mathrm{p}}^{\mu \nu} \frac{\delta^{(4)}(x-z)}{\sqrt{-g}}-\nabla_{\rho} B_{\mathrm{p}}^{\mu \nu \rho} \frac{\delta^{(4)}(x-z)}{\sqrt{-g}}\right) .
\end{aligned}
$$

The string part of the stress-energy tensor is written in the single-pole approximation, in accordance with the assumed absence of spin in the string interior. The stress-energy conservation equation is to be solved using this form of the stress-energy tensor, and thus imposes restrictions on the unknown variables $B_{\mathrm{S}}^{\mu \nu}(\xi)$, $B_{\mathrm{p}}^{\mu \nu}(\lambda), B_{\mathrm{p}}^{\mu \nu \rho}(\lambda)$ and $z^{\mu}(\xi)$. The latter determines the world sheet $\mathcal{M}$ of the string by the parametric equations $x^{\mu}=z^{\mu}(\xi)$. The procedure for determining these variables is described in detail in [4], and yields the result for the form of the stress-energy tensor along with familiar world sheet equations:

$$
B_{\mathrm{s}}^{\mu \nu}=m^{a b} u_{a}^{\mu} u_{b}^{\nu}, \quad \nabla_{a}\left(m^{a b} u_{b}^{\mu}\right)=0 .
$$

Here $u_{a}^{\mu} \equiv \partial z^{\mu} / \partial \xi^{a}$ represent vectors tangent to the world sheet, while $m^{a b}(\xi)$ are the free parameters that determine the type of matter string is made of. Operator $\nabla_{a}$ is the total covariant derivative in the direction of $\xi^{a}$.

The particle part $T_{\mathrm{p}}^{\mu \nu}$ is constrained by the requirement that particle trajectories coincide with the string boundary. The resulting boundary conditions are reinterpreted as the particle equations of motion, along with equations that determine the form of the particle stress-energy tensor:

$$
\begin{aligned}
& B_{\mathrm{p}}^{\mu \nu}=m v^{\mu} v^{\nu}, \quad B_{\mathrm{p}}^{\mu \nu \rho}=2 v^{(\mu} S^{\nu) \rho}, \\
& p_{\perp \lambda}^{\mu} p_{\perp \rho}{ }_{\nu} \frac{D S^{\lambda \rho}}{d s}=0, \\
& \frac{D}{d s}\left(m v^{\mu}\right)=\frac{D}{d s}\left(2 v_{\nu} \frac{D S^{\mu \nu}}{d s}\right)+v^{\nu} S^{\lambda \rho} R_{\nu \lambda \rho}^{\mu}+n_{a} m^{a b} u_{b}^{\mu} .
\end{aligned}
$$

Notation is the following. The world sheet boundary $\partial \mathcal{M}$ is typically consisted of two disjoint world lines, which we choose to parametrize with proper length parameter $s$. In other words, we fix the gauge $h_{i j}=-1$ and rename the parameter $\lambda$ to $s$. Given that, $v^{\mu} \equiv d z^{\mu} / d s$ is the world line tangent vector, while $p_{\perp}^{\mu} \equiv \delta_{\nu}^{\mu}+v^{\mu} v_{\nu}$ is the orthogonal projector to it. $n_{a}$ is a unit vector lying in $\mathcal{M}$ but orthogonal to $\partial \mathcal{M}$. The derivative $D / d s$ represents the covariant derivative in the direction of the world line, $D / d s \equiv v^{\mu} \nabla_{\mu}$. As for the string, so also here $m(s)$ and $S^{\mu \nu}(s)$ represent free parameters that determine the type of matter particle is made of. They have physical interpretation of particle mass and intrinsic angular momentum. If the boundary $\partial \mathcal{M}$ consists of two disjoint lines (as is typically the case), the mass and spin of the two particles are independent and so possibly distinct parameters for each line.

The boundary condition (3) represents the equation determining particle trajectory, and has three terms on the right-hand side, representing forces that act on the particle. The first term is the spin-orbit interaction term which represents the interaction of the rotation of the string with the spin of the particle. The second term is the familiar Papapetrou term representing deviation from a geodesic line for a spinning particle, and is the consequence of the interaction of particle angular momentum with the background gravitational field. The third term represents the force that the string exerts on the particle.

In what follows, we shall assume that the string is made of the Nambu-Goto type of matter, moving in flat spacetime:

$$
m^{a b}=T \gamma^{a b}, \quad R_{\nu \lambda \rho}^{\mu}=0 .
$$


Then, the world sheet equations (1) reduce to the familiar Nambu-Goto equations, and the third term on the right-hand side of (3) becomes $T n^{\mu}$ (here $n^{\mu} \equiv n^{a} u_{a}^{\mu}$ ). As for the particles, we shall impose the constraint

$$
S^{\mu \nu} v_{\nu}=0
$$

which rules out the boost degrees of freedom. Physically, this condition constrains the particle centre of mass to coincide with the string end (for the definition of centre of mass line and clarification of this constraint, see [4]). After this, we are left with

$$
\vec{S} \equiv \varepsilon^{\lambda \rho \mu 0} S_{\lambda \rho} \vec{e}_{\mu}
$$

as the only independent components of $S^{\mu \nu}$. Here $\vec{e}_{\mu}$ is a set of some orthonormal basis vectors in spacetime, $\vec{e}_{0}$ being timelike.

Now, we look for a simple, straight line solution of the equations of motion (1). Without loss of generality, we put

$$
\vec{z}=\vec{\alpha}(\tau) \sigma, \quad z^{0}=\tau,
$$

where $\xi^{0} \equiv \tau$ and $\xi^{1} \equiv \sigma$ take values in the intervals $(-\infty, \infty)$ and $[-1,1]$, respectively. Assuming that the string length $L=2|\vec{\alpha}|$, and the velocity of the string ends $V=|d \vec{\alpha} / d \tau|$ are constant, the equation (1) reduces to

$$
\frac{d^{2}}{d \tau^{2}} \vec{\alpha}+\omega^{2} \vec{\alpha}=0, \quad \omega \equiv \frac{2 V}{L} .
$$

It describes uniform rotation in a plane. Choosing the rotation plane to be the $x-y$ plane, we get the solution

$$
\vec{\alpha}=\frac{L}{2}\left(\cos \omega \tau \vec{e}_{x}+\sin \omega \tau \vec{e}_{y}\right) .
$$

Next we consider the boundary equations (2) and (3). Omitting the details of the calculation, we find that the particle intrinsic angular momentum satisfies

$$
\frac{d \vec{S}}{d \tau}=0, \quad \vec{S}=S \vec{e}_{z},
$$

while its velocity becomes

$$
V=\frac{1}{\sqrt{1+\frac{2 \mu}{T L}}}, \quad \mu \equiv m+\sqrt{\frac{2 T}{m L}} S .
$$

Each of the two particles has its own mass and intrinsic angular momentum, denoted by $m_{ \pm}$and $S_{ \pm}$for the particle at $\sigma= \pm 1$. As both particles have the same velocity, their masses are related by $\mu_{+}=\mu_{-} \equiv \mu$. We see that the particle masses $m_{ \pm}$may differ, in spite of the fact that the centre of mass of the stringparticle system is at $\sigma=0$. This is a consequence of the nontrivial spin-orbit interaction that contributes to the total energy.

By inspecting the expression (4), we see that $V<1$, as it should be. In the limit $\mu \rightarrow 0$, the string ends move with the speed of light, representing the Nambu-Goto dynamics with Neumann boundary conditions. When $\mu \rightarrow \infty$, the string ends do not move. This is an example of Dirichlet boundary conditions. 


\section{Regge trajectories law}

The total angular momentum and energy of the considered system are calculated using the usual definitions:

$$
E=\int d^{3} x T^{00}, \quad J^{\mu \nu}=\int d^{3} x x^{[\mu} T^{\nu] 0} .
$$

One finds

$$
\begin{aligned}
& E=T L \frac{\arcsin V}{V}+\frac{2 \mu}{\sqrt{1-V^{2}}}-\frac{2 V}{L}\left(S_{+}+S_{-}\right), \\
& J=\frac{T L^{2}}{4}\left(\frac{\arcsin V}{V^{2}}-\frac{\sqrt{1-V^{2}}}{V}\right)+\frac{2 \mu}{\sqrt{1-V^{2}}} \frac{L V}{2}+S_{+}+S_{-} .
\end{aligned}
$$

These equations have obvious interpretation. The total energy of the system consists of the string energy, kinetic energy of the two particles, and the spin-orbit interaction energy. The particle intrinsic rotational energy, being quadratic in $\vec{S}$, is neglected in the pole-dipole approximation. The total angular momentum includes the orbital angular momentum of the string and the two particles, and the particle spins.

In the limit of small particle masses, the free parameter $L$ can be eliminated in favour of $E$, which leads to

$$
J=\frac{1}{2 \pi T} E^{2}+2\left(S_{+}+S_{-}\right) .
$$

The first term on the right-hand side defines the known Regge trajectory, while the second represents a small correction due to the presence of spinning particles at the string ends. As we can see, the unique Regge trajectory of the ordinary string theory splits into a family of distinctive trajectories. It is interesting that the nonzero (but small) masses of the two particles do not contribute explicitly in the equation. They are present through the total energy $E$ of the system, but they do not bring any correction to the usual $J \propto E^{2}$ Regge law. In contrast, the spins of the particles, while also present implicitly through total angular momentum $J$, do give an explicit correction to the law, in the form of the second term on the right-hand side of (5).

\section{Concluding remarks}

In this paper we have analyzed the system consisting of a string with two particles attached to its ends. The method we use is a generalization of the Mathisson-Papapetrou method for pointlike matter [5, 6]. It has already been used in [3, 4] for the derivation of equations of motion of extended objects. Using those results, we have derived the equations of motion for the string along with the appropriate boundary conditions. These boundary conditions turn out to be the equations of motion for the two particles attached to the string ends.

These equations of motion display three forces acting on the particle. The first one is the spin-orbit interaction between the spin of the particle and its orbital motion due to the presence of the string. The second force represents geodesic deviation term due to the interaction of the particle spin with the background curvature. The third force acting on the particle is the pull of the string, since the particle is required to be on its end.

Next we specialized to the case of the usual Nambu-Goto string with two massive spinning particles at its ends. The equations of motion can be solved exactly for the case of a straight line string rotating around its center. It turns out that the velocity of the string ends is less than the velocity of light, and is dependent on the masses and spins of the particles. In this way, one is provided a way to describe both Neumann and Dirichlet boundary conditions for the Nambu-Goto string as the limits where the masses of the two particles approach zero or infinity, respectively. 
Finally, given this solution, one can calculate the total energy and angular momentum of the system, and in the limit of small particle masses derive the relation connecting the total angular momentum with the total energy and spins of the particles. This relation represents the law of Regge trajectories, with a correction term due to the particle spins. In this setting, there is not only one Regge trajectory, but a whole family, due to different spins of the constituent particles.

Acknowledgements This work was supported by the Serbian Science Foundation, Serbia.

\section{References}

[1] Y. Nambu, Phys. Rev. D 10, 4262 (1974).

[2] T. Goto, Prog. Theor. Phys. 46, 1560 (1971).

[3] M. Vasilić and M. Vojinović, Phys. Rev. D 73, 124013 (2006).

[4] M. Vasilić and M. Vojinović, JHEP 07 (2007) 028.

[5] M. Mathisson, Acta Phys. Polon. 6, 163 (1937).

[6] A. Papapetrou, Proc. R. Soc. A 209, 248 (1951). 